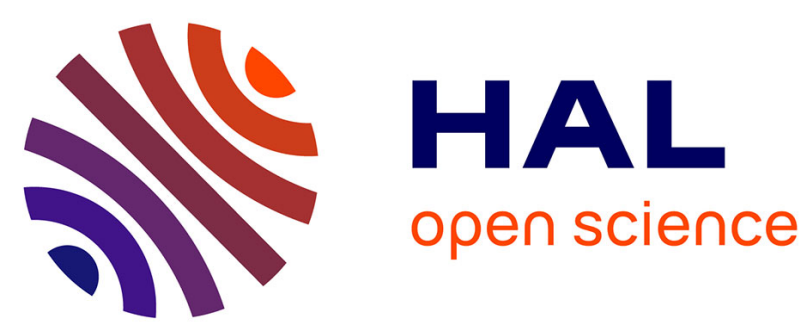

\title{
Ligand-Core NLO-Phores
}

Rodolphe Antoine

\section{To cite this version:}

Rodolphe Antoine. Ligand-Core NLO-Phores. Molecular Spectroscopy-Experiment and Theory, 26, Springer, Cham, pp.139-160, 2019, 978-3-030-01354-7. 10.1007/978-3-030-01355-4_5 . hal-02978501

\section{HAL Id: hal-02978501 \\ https://hal.science/hal-02978501}

Submitted on 31 Oct 2020

HAL is a multi-disciplinary open access archive for the deposit and dissemination of scientific research documents, whether they are published or not. The documents may come from teaching and research institutions in France or abroad, or from public or private research centers.
L'archive ouverte pluridisciplinaire HAL, est destinée au dépôt et à la diffusion de documents scientifiques de niveau recherche, publiés ou non, émanant des établissements d'enseignement et de recherche français ou étrangers, des laboratoires publics ou privés. 
Book: Molecular Spectroscopy - Experiment and Theory: From Molecules to Functional Materials Editors: Professor Andrzej Koleżyński and Professor Magdalena Król

Book series: Challenges and Advances in Computational Chemistry and Physics.

Publisher: Springer

Book Chapter :

Ligand-core NLO-phores. Two-photon absorption and two-photon

excited emission properties of atomically precise clusters of gold and silver.

Rodolphe Antoine

Institut lumière matière, UMR5306 Université Claude Bernard Lyon1-CNRS, Université de Lyon 69622 Villeurbanne cedex, France.

Email : rodolphe.antoine@ univ-lyon1.fr

\begin{abstract}
Functional ligand-protected noble metal cluster nanomaterials with enhanced two-photon absorption and two-photon excited emission may lead to new technologies in the fields of bio-imaging applications. In this article, we review experimental and theoretical methodologies allowing detailed investigation and analysis of two-photon absorption/emission properties of ligand-protected silver and gold metal clusters, coined "ligand-core" NLOphores. This includes femtosecond two-photon excited fluorescence experimental setups and quantum-chemical methodologies based on time-dependent density functional theory. We thoroughly analyze physical phenomena and trends leading to large two-photon absorption/emission responses of a few series of model nanoclusters focusing on the effects of the relaxation pathways in the linear and nonlinear optical regime, as well as innovative strategies aiming at enhancing their two-photon emission responses.
\end{abstract}




\section{Introduction}

Recent developments in optical imaging techniques, in particular multi-photon excitation (MPE) microscopy that allows studies of biological interactions at a deep cellular level, has motivated intensive research in developing multi-photon absorption fluorophores. Biological tissues are optically transparent in the near-infrared region. Therefore, fluorophores that can absorb light in the NIR region by multi-photon absorption are particularly useful in bio-imaging. MPE microscopy enables molecular imaging by using either exogenous markers or endogenous signals. Endogenous non-linear optics (NLO) signals are generally weak, unspecific and usually require to improve the instruments detection threshold and to develop scientific innovations, such as endogenous fluorescent proteins[1] to be applicable to MPE microscopy. Likewise, exogenous markers like organic dyes have been synthesized for MPE microscopy but their use remains limited by their relatively low two-photon excited fluorescence cross sections in aqueous environments and their rapid photo-bleaching.[2] In addition, other probes have been proposed including quantum and polymer nanodots with broad absorption and discrete, tunable emission wavelengths withstanding photobleaching.[3] Preliminary studies have clearly demonstrated that noble metal nanoclusters (NCs), nanomaterial made of few to hundred gold or silver protected by ligand molecules exhibiting molecular-like properties, [4] could also provide a valuable route in the nonlinear optical regime and MPO in particular.[5]

Multi-photon optics stems from the nonlinear light-matter interaction and can be described by a medium polarization $P$, induced by an intense optical electric field $E$ as $P=\chi^{(1)} E+\chi^{(2)} E E+$ $\chi^{(3)} E E E+\cdots$, where $\chi^{(1)}$ is the linear susceptibility tensor representing effects such as linear absorption and refraction, $\chi^{(2)}$ is the second-order nonlinear optical susceptibility, $\chi^{(3)}$ is the thirdorder nonlinear susceptibility and so on. Second Harmonic Generation (SHG) is a second-order process whereas Two-Photon Excitation Fluorescence (TPEF) and Third Harmonic Generation (THG) are both third-order processes. The combination of the three processes intrinsically provides different contrasts for living matter microscopy (see Figure 1, left).[6] Of note, the use of THG in the higher NIR region $(1.3-1.7 \mu \mathrm{m})$ is one of the strategy of choice for non-linear imaging in scattering tissues.[7] 


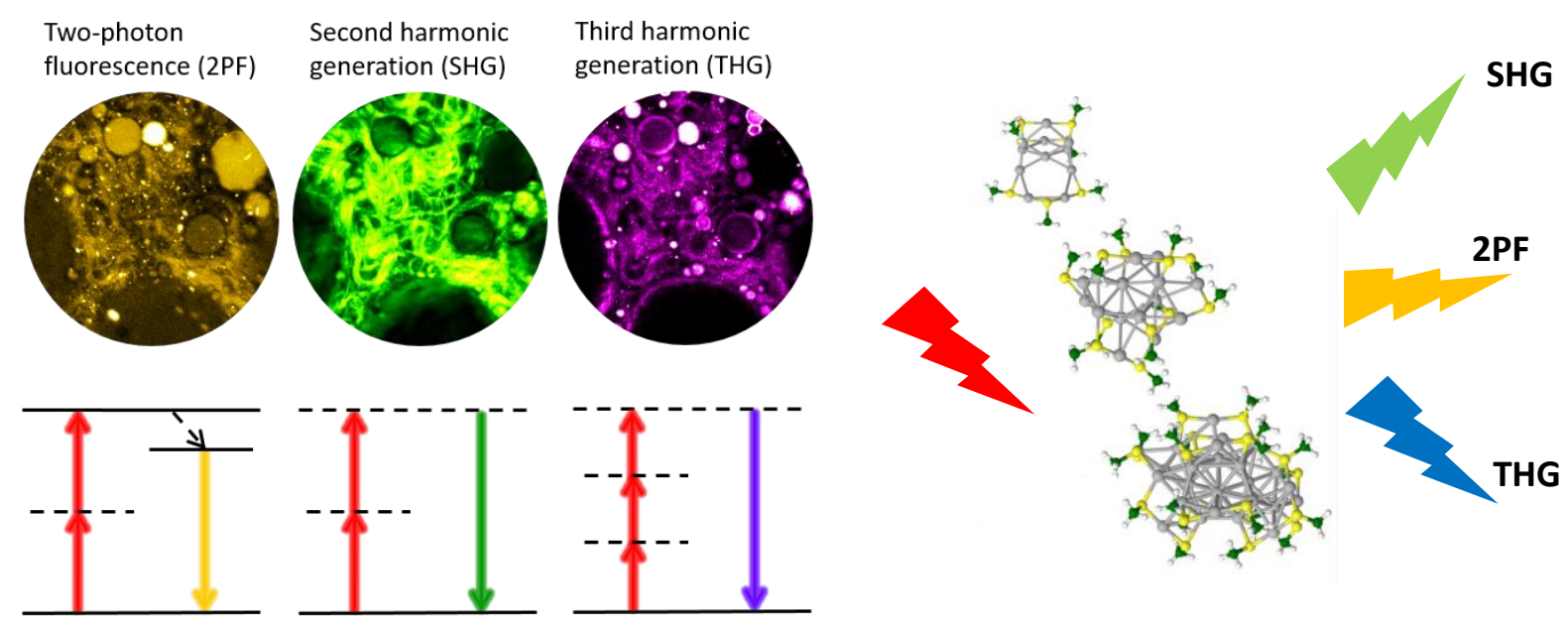

Figure 1: (left) Multicontrast images of an unperturbed human mammary tumor with various extracellular vesicles.

TPEF (2PF) are used to image fluorescent dyes or endogenous molecules. SHG is used to image noncentrosymmetric structures such as collagen fibers and THG is used to visualize the refractive index differences such as the interface of lipid droplets. (adapted from http://biophotonics.illinois.edu/imaging-technology/imagingtechniques/multiphoton-microscopy) (right) Selected protected metal clusters that may display different NLO signals.

Designing highly efficient second $\left(\chi^{(2)}\right)$ and third order $\left(\chi^{(3)}\right)$ NLO chromophores is largely a matter of finely combining a high density of delocalized electrons in a symmetrical or unsymmetrical environment. Gold and silver NCs constitute therefore good candidates (see fig. 1, right panel), although the reported $\left(\chi^{(2)}\right)$ values are still weaker than those of push-pull dyes[8] and are not currently competitive as contrast agents for MPO applications. Our theoretical and experimental joint investigation on ligand protected silver and gold clusters[9-16] has shown that the structure of the metal atom core, its charge and symmetry, dramatically influencing the NLO cross-sections and the core stabilizing ligands play a major role in NLO efficiencies. We coined this new class of NLO materials as "Ligand-Core" NLO-phores.[17,18]

This feature article overviews mainly experimental methodologies used for determination and analysis of two-photon absorption (TPA) and two-photon excited (TPEF) emission properties of atomically precise clusters of gold and silver. Using results obtained on atomically precise clusters of silver, we will show that both experimental and theoretical data can be used to get an extensive comprehension of the physics underlying the two-photon process and its amplitude, as well as to suggest an exploratory root for novel molecular engineering for further enhancement of TPA.[19] Also, we will describe some strategies that have been suggested recently to enhance TPEF properties. This includes the possibility to increase rigidity of the protective shell[20] as well as metal core-doping,[21] a promising strategy as it distorts the metallic atom core. 
The paper is organized as follows. In section 2 we define the atomically precise clusters of gold and silver, from their synthesis and characterization towards their (linear) optical properties. In section 3 we summarize the main theoretical and computational models used for calculation of the nonlinear responses and describe the leading factors responsible for enhanced NLO efficiencies. Section 4 deals with the principal experimental techniques and challenges for measurement of the TPA/TPEF cross section. A variety of representative "ligand-core" NLO-phores are investigated in section 5, allowing for rationalization of different structural effects on the TPA/TPEF cross section. Finally, the main conclusions as well as some future perspectives are drawn in section 6 .

\section{Atomically precise clusters of gold and silver: Synthesis, characterization and optical properties.}

\subsection{Atomically precise clusters of gold and silver.}

For small noble metal clusters -in the size range where each atom counts-, Mie-Drude-like model,[22] that predicts the optical response of free-electron metals in the bulk state, is no longer appropriate to discuss absorption spectra in the details. The geometry of the clusters must be determined by quantum chemistry methods that often use group theory and the optic response are described in terms of molecular transitions whose positions and intensities are predicted by sophisticated calculations of quantum mechanics.[19] As a pioneering work, the absorption spectra computed by equation-ofmotion coupled cluster singles and doubles (EOM-CCSD) and similarity transformed equation-ofmotion coupled cluster singles and doubles (STEOM-CCSD) methods with all-electrons basis sets, for the most stable structures of $\mathrm{Ag}_{5-8}$, nicely illustrated the molecular-like behavior of nanoclusters leading to an electronic energy quantization and the changes in the leading features of the patterns as a function of the cluster size.[23] While the optical properties of such small metal clusters have been largely investigated in the gas phase, their study in the solid and liquid phase requires some "stabilization" that prevents them from fragmentation or degradation. The use of solid gas or inorganic matrices permits to protect gold or silver clusters from photodissociation.[24-27] Organic scaffolds allow the formation and stabilization of metal clusters in solution. The use of organic scaffolds for fluorescent metal nanoclusters are relatively new, first reported by the group of Dickson in 2002 for silver nanoclusters.[28] These organic scaffolds have tremendous potentials, as the interaction between the ligands and metal clusters can be adjusted leading to tunability in their spectroscopic properties. For example by using DNA oligomers as organic scaffolds and by playing with the nucleotide sequence of DNA oligomers it is possible to synthesize silver nanoclusters that emit from the blue to near-infrared region.[29] 


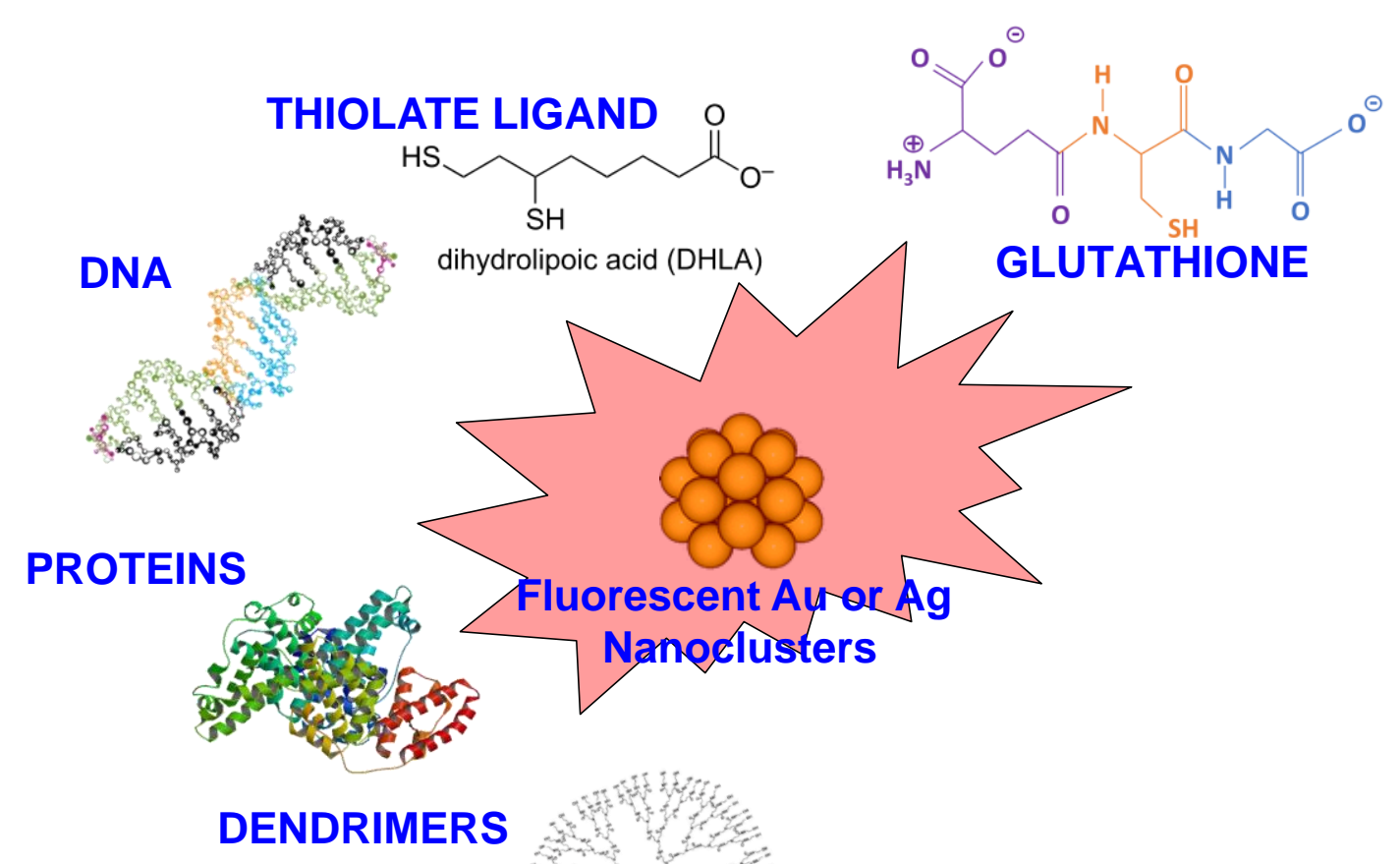

Figure 2: protected metal nanoclusters with possible scaffolds.

Ligands play very important roles in the formation of NCs as protective agents, which can prevent the metal clusters from aggregation and then keep the size-dependent fluorescence property. The formation and stabilization of gold or silver nanoclusters in solution have been accomplished in various ways (see Fig. 2). The proper choice of parameters for the reaction, including the temperature, the reducing method, the stabilizers and the initial ratio of metal salt:stabilizer, plays a crucial role for the successful synthesis of nanoclusters and to limit the size to few-atom nanoclusters. In addition to the ultrasmall size, the ligands used for NCs preparation also have impacts on their fluorescence properties. Jin and coworkers[30] demonstrated that for gold NCs, the surface ligands of NCs not only can be used as capping agent but also largely affect the fluorescence of NCs through charge transfer from surface ligand to the gold core. When the surface ligands have strong electron donation capability, the fluorescence can be enhanced. And the ligands with electron-rich atoms or groups have been found as a very effective choice for promising surface ligand of NCs to enhance the fluorescence.

\subsection{Atomically precise clusters of gold and silver. Synthetic routes}

The production of silver and gold nanoclusters can be performed following several routes. The metal ions from dissolved metal salts can be reduced, either by a chemical reductant (e.g. sodium borohydride, ...), (see fig. 3) by light (photoreduction with near-ultraviolet light) or by $\gamma$-rays (by radiolysis of water). The chemical reduction and the photoreduction are the most commonly used 
methods.[31] The specific properties of metal nanoclusters, such as the composition, stability and fluorescence quantum yield, depend largely on the scaffold used during reduction.

Thiols are frequently used on noble metal substrates because of the strong affinity of sulfur for these metals. And thiolated ligands (-SR) have appeared to be extremely good candidates to produce ultrasmall nanocluster sizes, in particular for gold.[32] Following the pioneering work of Brust et al.[33] based on the reduction of the metal precursors and the formation of metal core, thiol-containing small molecules were extensively used to stabilize gold and silver nanoclusters in the aqueous solution.[34,35] The use of thiol-containing small molecules as stabilizers permits to better control the production of gold NCs (AuNCs) than phosphine-capped ones, contributing to the stronger Au-S covalent bonding. Generally, the method of synthesizing thiolate-capped AuNCs has processes as follows. Gold salts $\left[\mathrm{AuCl}_{4}\right]^{-}$are dissolved in water and then transferred to an organic solvent by phase transfer agent; the thiols are added to the mixture inducing reduction of $\mathrm{Au}^{3+}$ ions into $\mathrm{Au}^{+}$ions and form $\mathrm{Au}^{+}-\mathrm{SR}$ complexes or polymers; then the $\mathrm{Au}^{+}$polymers are reduced by adding the reducing agent and lead to thiolate-protective gold nanoclusters.

Glutathione (GSH), a ubiquitous low-molecular weight thiol, played a significant role in producing gold NCs which showed good water solubility, bioactive surface, and high stability. Whetten and coworkers have proposed an unprecedented thiol-protective AuNC route by using the GSH (N- $\gamma$ glutamyl-cysteinyl-glycine) as the stabilizer. The as-synthesized AuNCs were fractionated by using polyacrylamide gel electrophoresis (PAGE) and characterized by mass spectrometry (MS).[36] Tsukuda and colleagues have also reported the characterization of fractionated AuNCs protected by GSH monolayers. The as-prepared AuNCs were isolated into single-sized Aun(SR)m clusters by the PAGE method and characterized using electrospray mass spectrometry.[37,38] Then, Bigioni et al. bridged the gap to use glutathione as a ligand to produce protected AgNCs that were isolated into single-sized Agn(SR)m clusters by the PAGE method and characterized using electrospray mass spectrometry.[39]

While the routes for producing metal NCs lead to a mixture of $\operatorname{Aun}(\mathrm{SR}) \mathrm{m}(\mathrm{Agn}(\mathrm{SR}) \mathrm{m})[40]$ cluster size, achieving atomic precision and molecular purity is challenging because the nanocluster growth is extremely complicated and remains poorly understood. Nevertheless, a systematic methodology called "size focusing", for achieving atomically precise clusters of gold and silver with molecular purity has been proposed. This methodology consists of two primary steps, (i) kinetically controlled synthesis of an $\mathrm{Mn}(\mathrm{SR}) \mathrm{m}$ mixture with a properly controlled size range and (ii) thermodynamically dictated sizefocusing of the mixture to single-sized nanoclusters. In parallel, a new approach, which is to utilize ligand exchange to induce size and structure transformation and, hence, to attain new $\mathrm{Mn}(\mathrm{SR}) \mathrm{m}$ nanoclusters was also proposed.[41] In parallel to these wild developed syntheses methods, Xie and co-workers proposed a kinetically controlled route utilizing carbon monoxide $(\mathrm{CO})$ as a mild reducing agent for the synthesis of size-selected Au NCs.[42] 
Concerning the characterization techniques, X-ray crystallography is of course the "holy-grail" technique to solve the crystal structure of nanoclusters and reveals the nature of bonding and the packing of atoms.[43-45,4] Recently, complete structures have been experimentally resolved for many gold and silver nanoclusters, including the most popular $\mathrm{Au}_{25}(\mathrm{SR})_{18}, \mathrm{Au}_{38}(\mathrm{SR})_{24}$, and $\mathrm{Au}_{102}(\mathrm{SR})_{44} \mathrm{NCs}$ by X-ray crystallography.[4] Many other methods are used to characterize noble metal NCs. X-ray photoelectron spectroscopy (XPS) is a valuable tool for confirming the oxidation state of gold in the sample, based on the Au-Au and Au-S binding energies. Various imaging techniques are widely employed to determine nanoparticle size and sample dispersity, such as transmission electron microscopy (TEM) and atomic force microscopy (AFM). The composition of NCs is best resolved by high resolution mass spectrometry (MS). The most widely used ionization methods for NCs characterization by MS are matrix-assisted laser desorption ionization (MALDI) and electrospray ionization (ESI).[46-48]

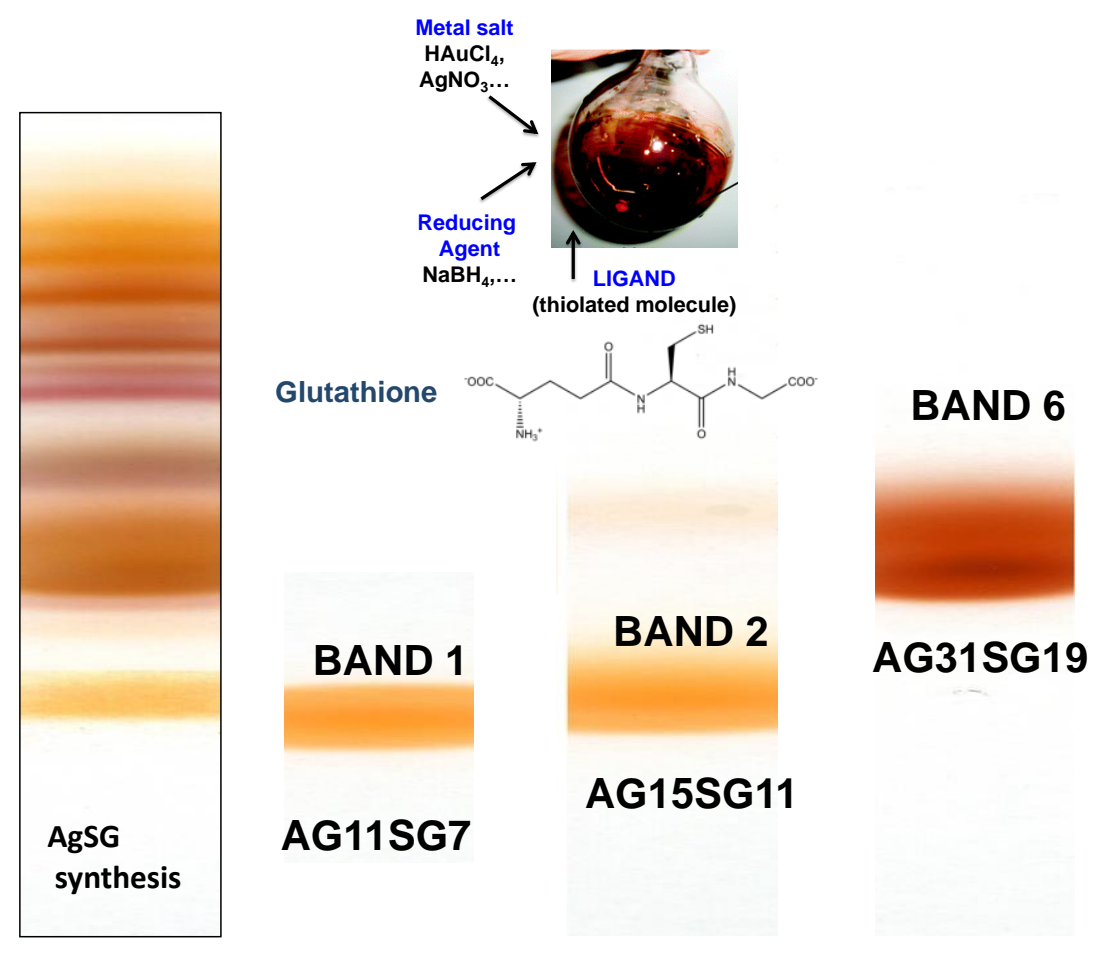

Figure 3: a) (top panel) General route to produce thiolate protected nanoclusters by a chemical reductant.(bottom panel) PAGE for Ag:SG clusters by (left) Bigioni synthesis (see ref.[39]) and (right) our focused Ag:SG synthesis. (see refs. [11,17])

\subsection{Atomically precise clusters of gold and silver. Optical properties.}

One-photon excited fluorescence (OPEF) of metal nanoparticles has of considerable interest due to their potential applications in biomedicine for instance. However, the origin and underlying 
mechanism of OPEF in these clusters is still poorly understood, although some recent theoretical investigations by Christine M. Aikens, using time-dependent density functional theory (TD-DFT), shed some new lights on the key processes for photon emission in such nanosystems.[49-51] Unravelling the crystal structure has permitted to better evaluate the structure-absorption relationships of some thiolate protected gold clusters. Time-dependent density functional theory (TDDFT) calculations on the electronic structure of $\mathrm{Au}_{25}$ clusters showed that the HOMO and the lowest three LUMOs are mainly composed of 6sp atomic orbitals of gold and a certain degree of the $S$ (3p).[52] The other higher HOMO orbitals are mainly constructed from the $5 \mathrm{~d}^{10}$ atomic orbitals of gold and hence constitute the d-band. The calculated absorption transitions are in agreement with the experimental observations. Recent ultrafast spectroscopic studies on relaxation of higher excited states have provided more insights into understanding the photoluminescence mechanism of $\mathrm{Au}_{25}$ clusters. Goodson et al. found that $500 \mathrm{~nm}$ emission fundamentally arises from the electron-hole recombination in the $\mathrm{Au}_{13}$ core with little perturbation from surface ligands, but NIR emission at $700 \mathrm{~nm}$ originates from the recombination of holes in the ground core state and electron decay from core excited states to $\mathrm{S}-\mathrm{Au}-\mathrm{S}-\mathrm{Au}-\mathrm{S}$ semi rings.[53] Jin et al., in a seminal work, found that charge state and surface ligands also have a significant influence on the NIR emission wavelength and quantum yields of $\mathrm{Au}_{25}$ clusters.[54] Clearly, more detailed photophysical studies are required for evaluating how surface ligands influence the photoluminescence mechanisms of such systems. The last ten years, some general trends have been figured out concerning the de-excitation pathways following a visible or near-UV absorption. The following experimental and theoretical findings were assembled from the present work and literature to derive the (very simplistic) energy diagram in Fig. 4.[55] Nearultraviolet and visible absorbance may arise from transitions between molecular orbitals with high ligand contribution to orbitals with high metal character (LMCT) and from metal-metal electronic transitions (LMMCT). A rapid ( $<1$ ps lifetime) decay pathway for clusters which have a core of metal atoms, that may lead to an emission in the visible. A long-lived (>100 ns lifetime), charge-transfer component is exhibited for all clusters. NIR emission in the clusters is related to the surface states and originates from the charge transfer excited state (see fig. 4). 


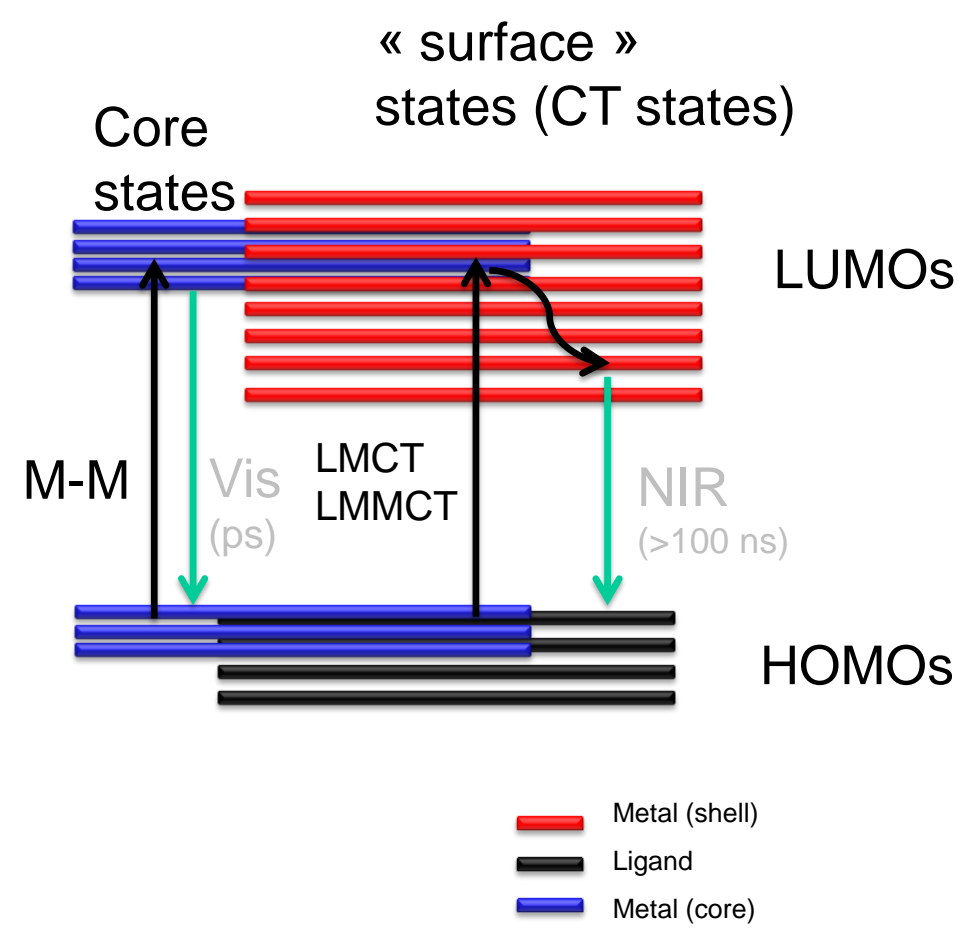

Figure 4: Cartoon diagram showing the relaxation pathways in gold nanoclusters following one photon absorption.

\section{Atomically precise clusters of gold and silver as new NLO chromophores: Background and design.}

The understanding of the structure-property relationship of molecular TPA is of great importance for the rational design of optimized two-photon chromophores. We will use the analogy with push-pull molecules in order to describe how theoretical data can be used to get an extensive comprehension of the physics underlying the two-photon process and its amplitude, as well as to suggest an exploratory root for novel chemical engineering for further enhancement of TPA in atomically precise clusters of silver and gold.

Push-pull dipolar molecules are characterized by a low-lying, high-intensity absorption band, related to the intramolecular charge transfer (ICT) between the electron donor (D) and acceptor (A) groups (see Figure 5). 

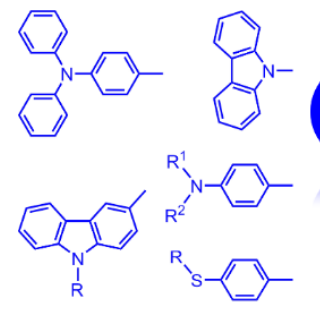

D

\section{$\pi$-conjugated system}
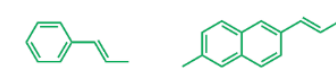
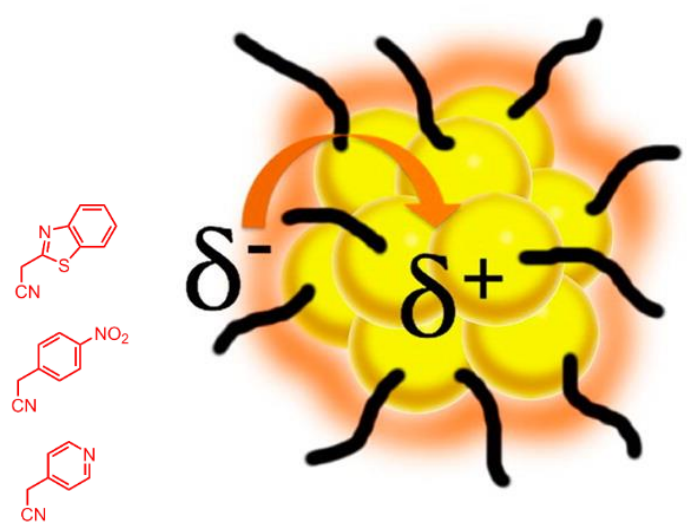

Figure 5: Schematic illustration of (left) a nonlinear optical (NLO), push-pull chromophore and (right) a "ligandcore" NLO-phore with ligand-protected silver and gold clusters.

The TPA cross section of such molecules is considered to be governed basically by two factors: transition dipole moments and transition energies of the molecule. As seen below, the theoretical expression of TPA cross section $\left(\delta_{\mathrm{TPA}}(\omega)\right)$ based on the perturbation expansion is comprised of the numerator including transition dipole moments and the denominator including the transition energies and the incident photon energy. The energy term governs the wavelength dispersion of $\delta_{\text {TPA }}(\omega)$ whereas the dipole moment term governs the overall magnitude of $\delta_{\mathrm{TPA}}(\omega)$. Thus, the structureproperty relationship for the molecules with large $\delta_{\mathrm{TPA}}(\omega)$ can be reduced by optimizing the transition dipole moments and frequencies involved in the TPA process.

It is commonly accepted that from the perturbation theory, the TPA cross section at the laser frequency of $\omega$ is given as [56]

$$
\delta_{T P A}(\omega)=\frac{(2 \pi)^{2} \omega^{2}}{(c h)^{2}} g(\omega)\left|S_{e g}\right|^{2}
$$

where $\mathrm{c}$ and $\mathrm{h}$ are the speed of light and the Planck constant, respectively. $g(\omega)$ denotes the normalized lineshape function of the TPA transition, $\left|S_{e g}\right|$ is so-called two-photon tensor.

The TPA cross section $\delta_{T P A}(\omega)$ can be written in SI units as :[57]

$$
\delta_{T P A}(\omega)=\frac{1}{5 c^{2} \hbar n^{2} \varepsilon_{0}^{2}} \frac{(\hbar \omega)^{2}}{\left(\hbar \omega_{e g}-\hbar \omega\right)^{2}+\Gamma_{e g}^{2}} \times\left[\frac{\left|\mu_{e g}\right|^{2}|\Delta \mu|^{2} \Gamma_{e g}}{\left(\hbar \omega_{e g}-2 \hbar \omega\right)^{2}+\Gamma_{e g}^{2}}+\frac{\left|\mu_{e g}\right|^{2}\left|\mu_{e e^{\prime}}\right|^{2} \Gamma_{e^{\prime} g}}{\left(\hbar \omega_{e^{\prime} g}-2 \hbar \omega\right)^{2}+\Gamma_{e^{\prime} g}^{2}}\right]
$$

The main parameters responsible for $\delta_{T P A}(\omega)$ are: change in the permanent dipole moment $\Delta \mu$; transition dipole moments $\mu_{e g}, \mu_{e e^{\prime}}$; angles between dipole moments; linewidth $\Gamma$, and detuning energies from intermediate and final states, $\left(\hbar \omega_{e g}-\hbar \omega\right)$ and $\left(\hbar \omega_{e^{\prime} g}-2 \hbar \omega\right)$. 
Enhancement of $\delta_{T P A}(\omega)$ can be obtained by playing with the following factors :

1. Increasing the transition dipole moments. In molecular design, this can be realized by increasing the $\pi$-conjugation length, or by introducing electron donor/acceptor groups. In noncentrosymmetric molecules, increasing the difference of the ground and excited state permanent dipole moments can also increase $\delta_{T P A}(\omega)$.

2. Maximizing resonance terms. Decreasing the detuning energy between intermediate and ground states can significantly enhance $\delta_{T P A}(\omega)$. If the intermediate state is located halfway between ground state and final state, a "double resonance" condition can be achieved, which can lead to a dramatic enhancement of $\delta_{T P A}(\omega)$. [58]

3. Reducing the linewidth of the lowest energy one-photon transition.

Such factors have been figured out in details for design strategies and structure-property relations of cyanine and cyanine-like molecular structures with the goal of enhancing TPA in the near-IR for multiphoton fluorescence sensing applications.[57]

Atomically precise nanoclusters of silver or gold can be viewed as a "multi-shell system" composed by (1) a metallic core, (2) a metal-ligand interface, in particular with staple motifs leading to metalsulfur bonds, and (3) the surface ligand molecules. These three shells may communicate in two different ways : charge transfer from ligand to metal core (analogy with ligand-to-metal charge transfer (LMCT) or ligand-to-metal-metal charge transfer (LMMCT) observed in metal complexes) and through direct bonding or direct donation of delocalized electrons of electron-rich groups of the ligands.[18] Such "communications" between ligands and metal core may increase the transition dipole moments leading to enhanced $\delta_{T P A}(\omega)$.

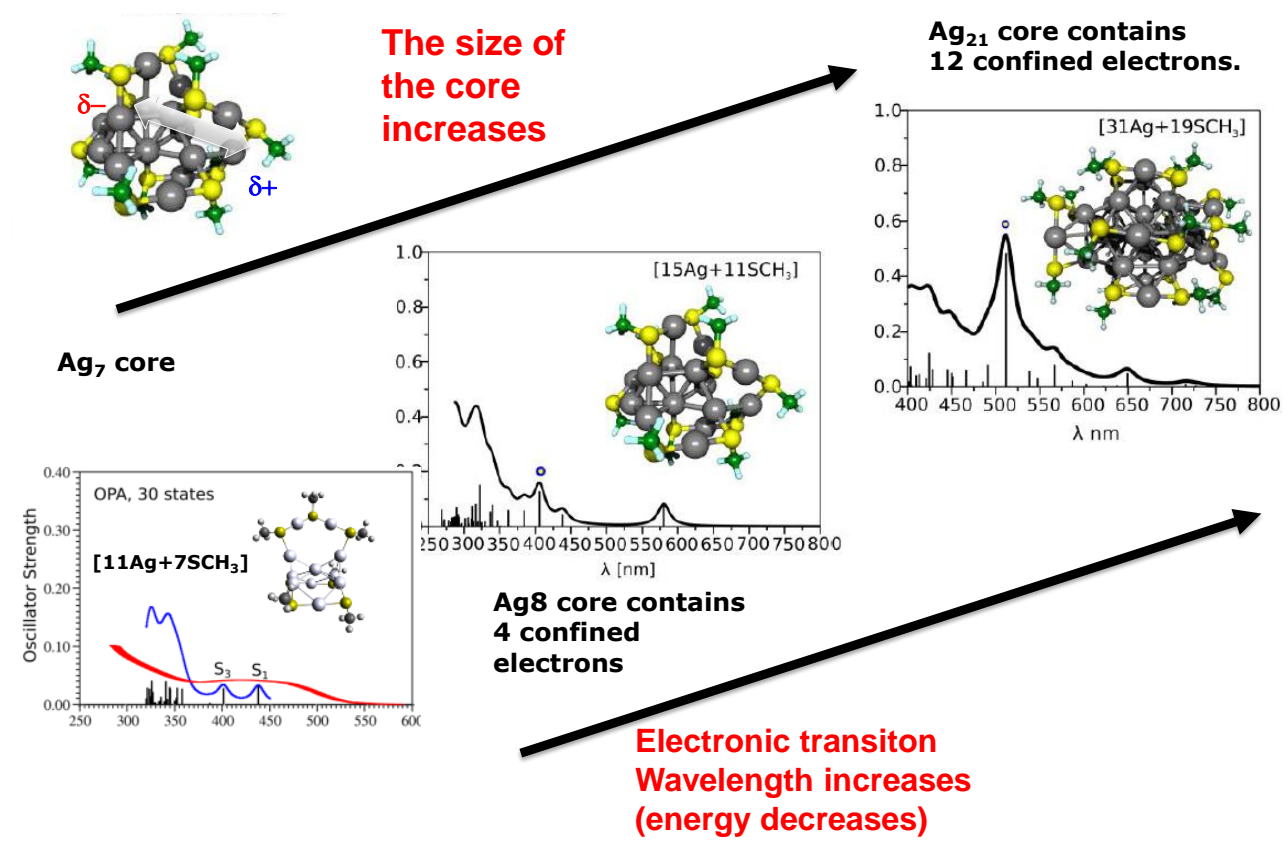

Figure 6: Comparison of TDDFT TPA spectra (left) of Ag11(SCH3)7, Ag15(SCH3)11 and Ag31(SCH3)19 
Density functional theory (DFT) and its time-dependent version (TDDFT) have been used for determination of the structural and optical properties of ligand-protected silver and gold clusters. There are two approaches for addressing two-photon absorption within analytic response method: First, the calculation of third-order frequency-dependent response function from the second hyperpolarizability in which imaginary part is related to TPA cross section; the second approach involves the single residue of the second-order response function or the first hyperpolarizability. The latter represents more practical way of computing the TPA cross section. In spite of the success, it is necessary to notice that in standard response theory, the response functions can diverge, since the response function has poles whenever one or more of the optical frequencies equal an excitation energy. This can lead to nonphysical behavior for molecular properties in the resonance region. However, introducing damping terms in different ways the singularities of the response functions can be corrected or effectively removed.[15]

The two-photon absorption cross section for an excitation from the ground state $\mid 0>$ to a final state $\mid f>$ is defined in terms of normalized shape function $g\left(\omega_{\mu}+\omega_{v}\right)$ and the the two-photon absorption transition amplitude tensor $\mathrm{T}^{\omega \mu \omega v, \mathrm{f}}$

$$
\sigma_{T P A}=\frac{(2 \pi e)^{4} \omega_{v} \omega_{\mu}}{c^{2}} g\left(\omega_{v}+\omega_{\mu}\right)\left|T^{\omega_{v} \omega_{\mu}, f}\right|^{2}
$$

In order to obtain this tensor, the quadratic density functional response theory was applied. Two-photon absorption transition amplitude tensor $\left|T^{\omega_{\nu} \omega_{\mu}, f}\right|^{2}$ between the ground and the excited state is defined as

$$
T_{a b}^{2 \omega, f}=\sum_{k}\left[\frac{\left\langle 0\left|\hat{\mu}_{a}\right| k\right\rangle\left\langle k\left|\hat{\mu}_{b}\right| f\right\rangle}{\omega_{k}-\omega_{f} / 2}+\frac{\left\langle 0\left|\hat{\mu}_{b}\right| k\right\rangle\left\langle k\left|\hat{\mu}_{a}\right| f\right\rangle}{\omega_{k}-\omega_{f} / 2}\right]
$$

where it is assumed that frequency of incident radiation is equal to half of excitation energy from ground to excited state, i.e. $\omega=\omega_{\mathrm{f}} / 2$. In the above equation $\mu_{\mathrm{a}}$ and $\mu_{\mathrm{b}}$ are Cartesian components of dipole moment operator $\mu$, and $\omega_{\mathrm{k}}$ and $\omega_{\mathrm{f}}$ are the frequencies of excitation from $\mid 0>$ to $\mid \mathrm{k}>$ and $|\mathrm{f}\rangle$ respectively. Thus application of this formula includes explicit summation over excited states and requires computation of dipole moment operator $\mu$ between excited states. Of note, in order to prevent the TPA cross sections from blowing up near the one- 
photon resonances, the SOSs approach uses a damping factor $\Gamma$. More details about computational details can be found in ref.[15,18]

We conducted a theoretical investigation of the nonlinear optical properties for the lowest energy structures of the $\mathrm{Ag}_{11} \mathrm{~L}_{7}, \mathrm{Ag}_{15} \mathrm{~L}_{11}$ and $\mathrm{Ag}_{31} \mathrm{~L}_{19}$ nanoclusters, where $\mathrm{L}$ stands for the $\mathrm{SCH}_{3}$ group (see fig. 6).[17] They contain respectively 4,8 and 12 delocalized electrons within the core. Several factors influencing the TPA cross-sections have been figure out: (i) the excitation between ligands and the metal core are characteristic of the nonlinear transitions, (ii) the "double resonance" between states involved in the OPA and TPA processes is required to obtain giant TPA cross-sections, (iii) large dipole transition moments are related to a non-uniform electron distribution within the metal core. The role of the structural properties, i.e. of the geometry of the metal core in determining this electron distribution, is therefore crucial.

In the case of the TPA cross-sections, interplay between a resonance effect and large transition dipole moments is essential. As the size of the silver nanoclusters increases, transition dipole moments should continue to increase due to larger core size (and thus larger core-to-ligand distances) and/or nonuniform electronic distribution in the metal core. Also, the optical band gap decreases as the size of the nanoclusters increases,[45] shifting the spectra towards the NIR spectral region, a feature useful for bio-imaging applications.

\section{Measurement Techniques of Optical Nonlinearities: Two-photon absorption/fluorescence.}



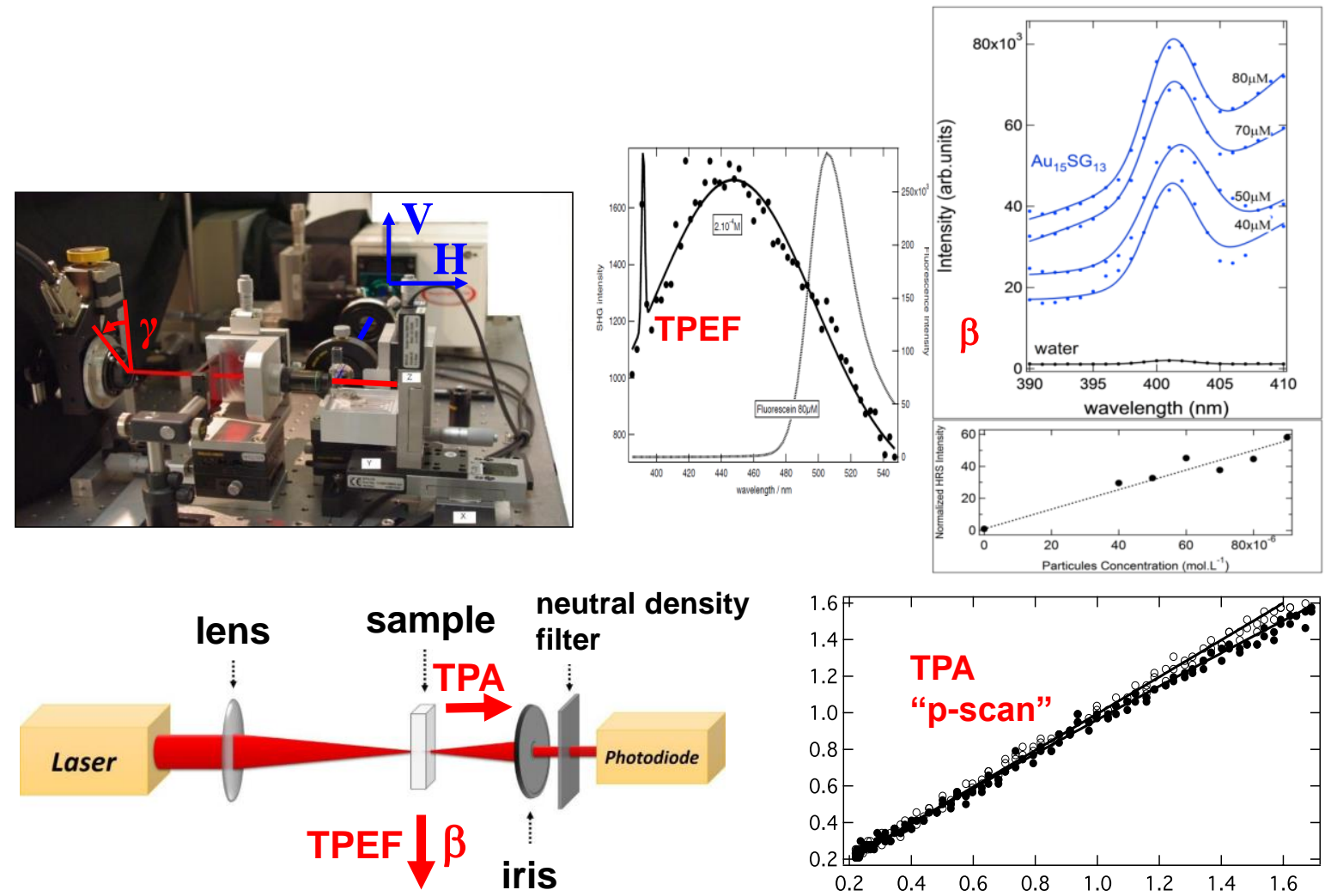

Figure 7: Schematic view and photo of the classical $90^{\circ}$ angle Hyper-Rayleigh scattering geometry. The set-up was recently upgraded in a "tout-en-un" experimental set-up able to record TPA, TPEF cross sections along with the first hyperpolarizability $\beta$ values for liganded silver and gold quantum clusters.

The light source used in our experimental device is a titanium-sapphire femtosecond laser tunable between 700 and $1000 \mathrm{~nm}$ (Coherent, Chameleon Ultra I) delivering 180 fs pulses duration with a repetition rate of $76 \mathrm{MHz}$. At the exit of this laser cavity, an optical system was inserted, composed of a half-wave plate, a polarizer cube allowing the control of the laser power.

The power-controlled laser beam is then directed to a second half-wave plate for polarization control thereof. A low-pass filter placed immediately after this second blade makes it possible to suppress the light generated at the harmonic frequency before the cell. The laser beam at the fundamental frequency is then focused in the cell whose walls are made of quartz in order to obtain an excellent transmission at the harmonic frequency around $400 \mathrm{~nm}$ by a microscope objective X10 with numerical aperture NA $=0.25$ (Thorlabs, LMH-10X-1064). The scattered intensity is then collected at right angles using a lens with a focal length of $50 \mathrm{~mm}$ and separated from the fundamental intensity scattered by a highpass filter. A polarization analysis system, consisting of a half wave plate and a polarizing cube adapted to the wavelength of $400 \mathrm{~nm}$, is placed on the detection line in order to select a polarization state defined by the diffused harmonic wave. The intensity at the harmonic frequency is then detected, using a photomultiplier tube placed at the output of a spectrometer (Jobin-Yvon, iHR320). 
A spectrometer makes it possible to choose the range of length of the collected signal and to obtain the TPEF spectrum or the SHG signal.

Recently, we developed a "tout-en-un" experimental set-up able to record TPA, TPEF cross sections along with the first hyperpolarizability $\beta$ values for liganded silver and gold quantum clusters.[59,12,60,61]

The light source for the present TPA experiments was the same as the above. The beam was gently focused by a $5 \mathrm{~cm}$ focal lens and sent in transmission into a $0.5 \mathrm{~cm}$ path length spectrophotometric cuvette. The transmitted light was detected with a large aperture photodiode. The incident power was controlled with a half-wave plate and a polarizing cube. The sample absorption was then determined as a function of the incident power. Fluorescein was used as a reference to determine the beam waist at focus in particular. The calibration of the photodiode signal was obtained with a neat water cuvette through a variation of the incident power. As expected with a distilled water cuvette, the plot of the transmitted light versus the incident light exhibits a linear behavior whereas in the presence of the nanoclusters, a decrease of the transmitted intensity is observed as the absorption increases due to the nonlinear contribution.

The transmission factor $T$ of the sample can then be expressed as a function of intensity using the standard Beer-Lambert law:

$T=C e^{-\alpha L}=C e^{-(\alpha+\delta I) L}$

where $C$ is a constant. . It is possible to extract $\delta$ from the slope of the $T\left(P_{m}\right)$ vs. $P_{m}$ dependence.

The TPE cross-sections of the silver or gold NC samples were calculated using fluorescein in aqueous solution as a reference (see fig. 7):

$\sigma_{T P E F}=\frac{\eta^{r e f} \sigma_{2}^{r e f} c^{r e f}}{c} \frac{I}{I^{r e f}}$

Here, the index ref denotes values related to the reference measurements. where $\sigma$ is the integrated area of the two-photon emission intensity of the sample and the reference ref, $\mathrm{C}^{\text {ref }}$ is the concentration of fluorescein and $\mathrm{C}$ the concentration of the NCs. For example, the TPE cross-section of reference (fluorescein) at $780 \mathrm{~nm}$ is $24 \mathrm{GM}$ according to previous reports.[62]

\section{Case studies.}

\subsection{NLO emission of Ag29(DHLA)12. Playing with photons and colors.}



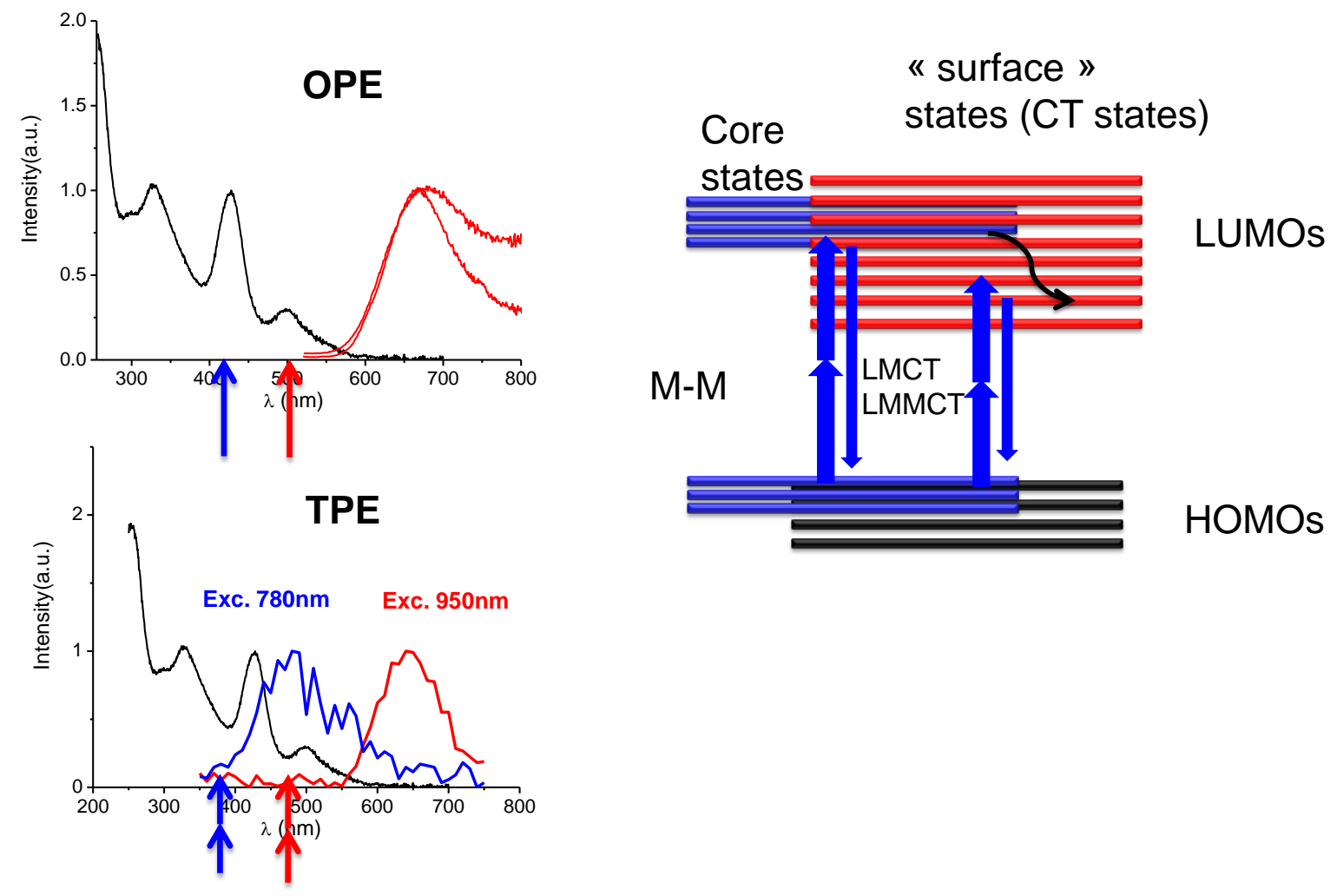

Figure 8: (left panel) Spectroscopic characterization of the synthesized Ag29(DHLA)12 clusters dispersed in water. Absorption spectrum (black), OPEF spectrum (top) and TPEF spectrum (bottom). The arrows represent the wavelength of excitation by OPE and TPE processes (right panel) Schematics showing the proposed excited state relaxation dynamics in the Ag29(DHLA)12 clusters in TPEF experiments.

As a first example, we investigated in details the nonlinear optical response of $\operatorname{Ag}_{29}$ (DHLA) $)_{12}$. The two-photon excited fluorescence spectra were recorded and their first hyperpolarizability obtained. A general observation: these NCs have huge two-photon absorption cross sections typically $510^{4} \mathrm{GM}^{1}$ for $\mathrm{Ag}_{29}$ while their corresponding two-photon excited fluorescence cross section is only 0.5 GM.[14] For NLO applications, they are still less effective than dyes, but usually these clusters present a good biocompatible and low toxicity. We have also observed for the $\operatorname{Ag}_{29}$ an interesting behavior for potential applications, which concerns its emission properties following the absorption of 1 or 2 photons, as shown in the figure 8 . With an excitation with one photon at $400 \mathrm{~nm}$ or by two photons at $800 \mathrm{~nm}$, the resulting emission spectrum is either blue or red (see fig. 8). Interestingly, this unique $\mathrm{Ag}_{29}$ nanocluster display two colors, playing with the number of photons involved in the excitation.

\footnotetext{
${ }^{1}$ Maria Goeppert-Mayer, Nobel Prize in Physics 1963. Her name is now known for the unit of the molecular TPA cross-section (GM) : $1 \mathrm{GM}=10^{-50} \mathrm{~cm}^{4}$.s. photons ${ }^{-1}$
} 
This behavior is still poorly understood and probably reflects the complexity of the relaxation of excited electronic states characteristic of the molecular or quantum regime.

For organic molecules, the emission typically occurs from its lowest excited energy level for a given spin multiplicity (referred to as Kasha's rule).[63] However nanoclusters as well as certain organic molecules tend to break this rule and emit at more than one possible wavelength depending on the electronic structure, density of states and excited state dynamics involved. Regardless of whether a molecule/nanocluster obeys the Kasha's rule or not, in order to gain a more detailed understanding of the excited state emission dynamics of a chromophore, one may carry out the time resolved experiments. Note that the photochemistry of gold nanoclusters is more complex than the de-excitation of $\pi-\pi^{*}$ states of organic molecules, where excitations within the gold core as well as couplings with surface states (through LMCT and LMMCT), singlet to triplet states conversion, may occur...

\subsection{Bulky counterions. A simple route to enhance the TPEF efficiencies.}

\section{At this stage, we reckon that protected gold quantum clusters are excellent two-photon} absorbers but rather poor two-photon excited emitters.[61] To enhance third order $\left(\chi^{(3)}\right)$ efficiencies, the ligand shell rigidity is an interesting strategy that would allow for enhanced photon emission as compared to non-radiative relaxation upon photo-excitation. Pyo et al.[20] have shown that it is possible to achieve OPEF quantum yield $>60 \%$ by rigidifying the metal-sulfur interface with the binding of bulky groups. We recently pushed forward this concept to the nonlinear optical regime.
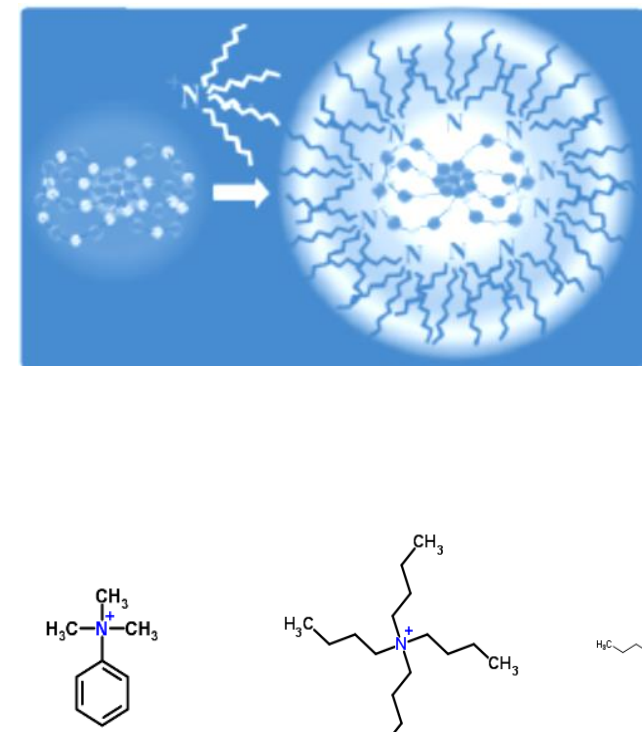

TMph : BzTMA

trimethylphenyl ammonium

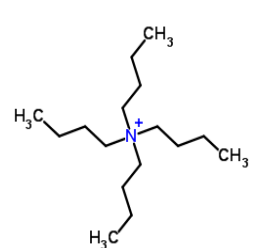

TBA :

Tetrabutyl ammonium

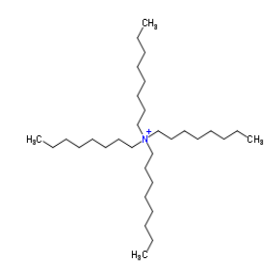

TOA :

Tetraoctyl ammonium

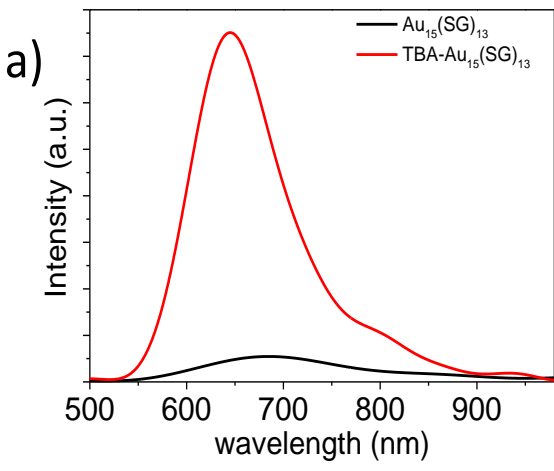

b)

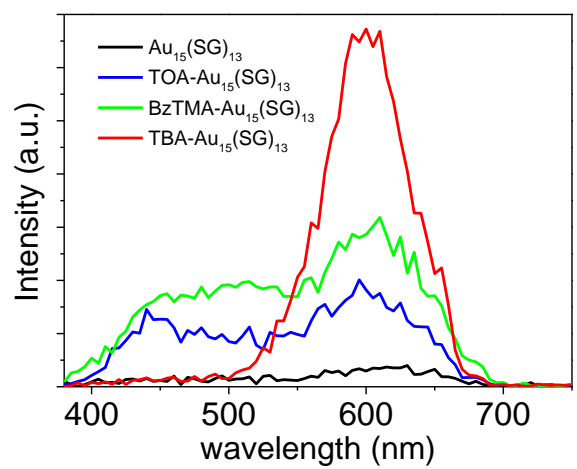


Figure 9: (left panels) Schematic and structure of bulky counterions used to bind to glutathione-protected gold clusters. (right panels) (top) One-photon excited fluorescence spectra of Au15(SG)13 in water and TBAAu15(SG)13 in methanol. (bottom) Two-photon excited fluorescence spectra at excitation wavelength $780 \mathrm{~nm}$ of Au15(SG)13 in aqueous solution compared to different bulky cations- Au15(SG)13 in methanol.

The strategy developed recently is to use bulky ammonium counterions, a nitrogen cation surrounded by 4 alkyl chains. In solution, the luminescence of the NCs is often affected by the solvent (in particular water). Counter-ions will interact with the NC surface by electrostatic interaction (between the counterions and the carboxylate groups of the glutathione ligands) to stick to the surface of the NCs which will have the effect of protecting it from the environment and also to "rigidify" its surface. The effect is spectacular on the 1-photon excited fluorescence spectra, as shown on $\mathrm{Au}_{15}$ in water. When such NCs are complexed with tetrabutylammonium ions, they become extremely fluorescent (see Fig. 9). We extended this study to the NLO regime and were able to show that the same dramatic effect is observed for TPEF and with a variety of bulky counterions; the best candidate to date being tetrabutylammonium (TBA). The TPEF cross sections of these objects then become interesting for multiphoton optics and we have bridged the gap towards 2-photon confocal microscopy.
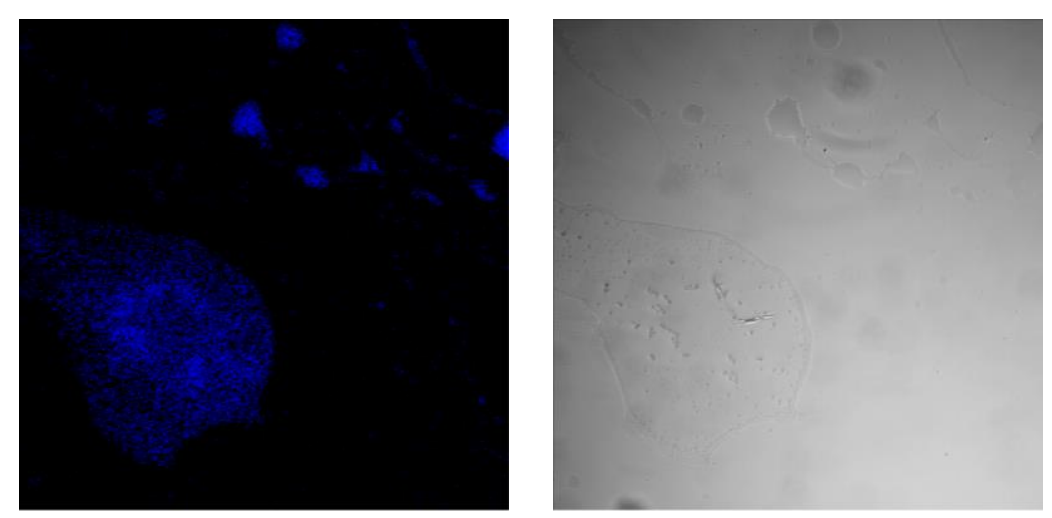

Figure 10: (left) TPEF intensity raster scan of Au NCs containing methanol droplets in heptane with (right) the corresponding optical image (size of the image : $637 \times 637 \mu \mathrm{m}$ ). 1 exc $=780 \mathrm{~nm}$.

TPEF measurements were made with a confocal microscope with $780 \mathrm{~nm}$ excitation from a focused fs laser in a small well containing the NCs solution. We carried out an XY mapping of the sample which allowed us to locate an interesting area and then a Z-scan with a motorized microscope objective. The emitted signal was collected in epifluorescence mode. As a proof of concept and before going to cell imaging, we made an emulsion from a methanol-heptane mixture, it gives rise to methanol droplets trapped in heptane of micrometric size. Au NCs in the presence of TBA counterions are soluble in 
water and were used to image the droplets. The topographic and TPEF images are given in Fig. 10, where we recognize the droplet containing the NCs.

\section{Conclusion and outlooks.}

The aim of the present study was to gain a fundamental knowledge on the mechanism involved in multiphoton processes in atomically precise gold and silver nanoclusters. By measuring two photon absorption, two photon excited fluorescence cross sections and first hyperpolarizability for such NCs, we have provided unique benchmarks for theoretical modeling of the origin of enhanced NLO properties of ultra-small ligated metal clusters, as well as the interplay between the cluster core and the interface between the ligand shell and the metallic part (ligand-core NLO-phores).

It will also serve as a basis for further developments in the design of high efficiency NLO-phores with good stability and low toxicity for in vitro (and possibly ultimately in vivo) bio-imaging applications. One of the technological objective of this study will be to bridge the gap between new cell biomarkers and 2-photon NLO-phores design.

Designing highly efficient second- and third-order nonlinear optical (NLO) chromophores is largely a matter of finely combining a high density of delocalized electrons in a symmetrical or unsymmetrical environment. For enhancing NLO efficiencies, innovative strategies should be explored in the future. To enhance second order $\left(\chi^{(2)}\right)$ efficiencies, metal core-doping is a promising strategy as it distorts the metallic atom core.[64] Also, enhanced fluorescence of AuNCs by silver doping was reported by Le Guével et al.[65]'[66] Therefore to enhance third order $\left(\chi^{(3)}\right)$ efficiencies, silver doping can be coupled to ligand shell rigidity as the latter favors the radiative properties of NCs. This route was recently explored by Olesiak-Banska et al. who showed that AuAgNCs exhibit two-photon excited luminescence (2PL) emission and second-harmonic generation (SHG) and that these properties remain the same in liquid crystalline matrix.[66] An alternative route to enhance third order $\left(\chi^{(3)}\right)$ efficiencies, is to use the concept of "rigidity". We recently pushed forward this strategy consisting in using bulky counterions to enhance the luminescence in the nonlinear optical regime. We showed that by an appropriate choice of bulky counterions and of solvent, a 30-fold increase in TPEF signal in the red for glutathione-protected gold clusters could be obtained. A simple way to increase rigidity of the protective shell is to replace thiolated ligands by proteins instead. Bovine serum albumin-Au25NCs indeed exhibit an efficient two-photon absorption followed by blue[67] to red to near-infrared photoluminescence.[68] Using (bio)organic thiolate templates, further enhancement can be achieved by the increasing rigidity of the metal-sulfur interface. The precise tailoring of the 
hydrophilicity/hydrophobicity balance on the gold or silver NC surfaces can be a way to increase the fluorescence signal.[69,70]

\section{Acknowledgements.}

Most of the work presented would not have been possible without the fruitful collaborations with Isabelle Russier-Antoine, Franck Bertorelle, Željka Sanader, Marjan Krstić, Philippe Dugourd, PierreFrançois Brevet and Vlasta Bonačić-Koutecký. Therefore, I wish to express my deepest gratitude to them. Furthermore, I would like to acknowledge financial support of the French-Croatian project "International Laboratory for Nano Clusters and Biological Aging, LIA NCBA".

\section{References.}

1. De Meulenaere E, Nguyen Bich N, de Wergifosse M, Van Hecke K, Van Meervelt L, Vanderleyden J, Champagne B, Clays K (2013) Improving the Second-Order Nonlinear Optical Response of Fluorescent Proteins: The Symmetry Argument. Journal of the American Chemical Society 135 (10):4061-4069. doi:10.1021/ja400098b

2. Terenziani F, Katan C, Badaeva E, Tretiak S, Blanchard-Desce M (2008) Enhanced Two-Photon Absorption of Organic Chromophores: Theoretical and Experimental Assessments. Advanced Materials 20 (24):4641-4678. doi:10.1002/adma.200800402

3. Medintz IL, Uyeda HT, Goldman ER, Mattoussi H (2005) Quantum dot bioconjugates for imaging, labelling and sensing. Nature Materials 4:435. doi:10.1038/nmat1390

4. Jin R, Zeng C, Zhou M, Chen Y (2016) Atomically Precise Colloidal Metal Nanoclusters and Nanoparticles: Fundamentals and Opportunities. Chemical Reviews 116 (18):10346-10413. doi:10.1021/acs.chemrev.5b00703

5. Polavarapu L, Manna M, Xu Q-H (2011) Biocompatible glutathione capped gold clusters as oneand two-photon excitation fluorescence contrast agents for live cells imaging. Nanoscale 3 (2):429434. doi:10.1039/c0nr00458h

6. Tu H, Liu Y, Marjanovic M, Chaney EJ, You S, Zhao Y, Boppart SA (2017) Concurrence of extracellular vesicle enrichment and metabolic switch visualized label-free in the tumor microenvironment. Science Advances 3 (1). doi:10.1126/sciadv. 1600675

7. Yue S, Slipchenko MN, Cheng JX (2011) Multimodal nonlinear optical microscopy. Laser \& Photonics Reviews 5 (4):496-512. doi:10.1002/Ipor.201000027

8. Blanchard-Desce M (2002) Molecular engineering of NLO-phores for new NLO microscopies. Comptes Rendus Physique 3 (4):439-448. doi:https://doi.org/10.1016/S1631-0705(02)01329-4

9. Bellina B, Antoine R, Broyer M, Gell L, Sanader Z, Mitric R, Bonacic-Koutecky V, Dugourd P (2013) Formation and characterization of thioglycolic acid-silver cluster complexes. Dalton Transactions 42 (23):8328-8333

10. Bellina B, Compagnon I, Bertorelle F, Broyer M, Antoine R, Dugourd P, Gell L, Kulesza A, Mitric R, Bonacic-Koutecky V (2011) Structural and Optical Properties of Isolated Noble Metal-Glutathione Complexes: Insight into the Chemistry of Liganded Nanoclusters. Journal of Physical Chemistry C 115:24549-24554

11. Bertorelle F, Hamouda R, Rayane D, Broyer M, Antoine R, Dugourd P, Gell L, Kulesza A, Mitric R, Bonacic-Koutecky V (2013) Synthesis, characterization and optical properties of low nuclearity liganded silver clusters: Ag31(SG)19 and Ag15(SG)11. Nanoscale 5 (12):5637-5643

12. Bertorelle F, Russier-Antoine I, Calin N, Comby-Zerbino C, Bensalah-Ledoux A, Guy S, Dugourd P, Brevet P-F, Sanader Ž, Krstić M, Bonačić-Koutecký V, Antoine R (2017) Au10(SG)10: A Chiral Gold Catenane Nanocluster with Zero Confined Electrons. Optical Properties and First-Principles 
Theoretical Analysis. The Journal of Physical Chemistry Letters:1979-1985.

doi:10.1021/acs.jpclett.7b00611

13. Bonacic-Koutecky V, Kulesza A, Gell L, Mitric R, Antoine R, Bertorelle F, Hamouda R, Rayane D, Broyer M, Tabarin T, Dugourd P (2012) Silver cluster-biomolecule hybrids: from basics towards sensors / perspective article. Physical Chemistry Chemical Physics 14:9282-9290

14. Russier-Antoine I, Bertorelle F, Hamouda R, Rayane D, Dugourd P, Sanader Z, Bonacic-Koutecky V, Brevet P-F, Antoine R (2016) Tuning Ag29 nanocluster light emission from red to blue with one and two-photon excitation. Nanoscale 8 (5):2892-2898. doi:10.1039/C5NR08122J

15. Sanader Z, Krstic M, Russier-Antoine I, Bertorelle F, Dugourd P, Brevet P-F, Antoine R, BonacicKoutecky V (2016) Two-photon absorption of ligand-protected Ag15 nanoclusters. Towards a new class of nonlinear optics nanomaterials. Physical Chemistry Chemical Physics 18 (18):12404-12408. doi:10.1039/C6CP00207B

16. Sanader Z, Mitric R, Bonacic-Koutecky V, Bellina B, Antoine R, Dugourd P (2014) Nature of electronic excitations at the metal-bioorganic interface illustrated on histidine-silver hybrids. Physical Chemistry Chemical Physics 16:1257-1261

17. Russier-Antoine I, Bertorelle F, Calin N, Sanader Z, Krstic M, Comby-Zerbino C, Dugourd P, Brevet P-F, Bonacic-Koutecky V, Antoine R (2017) Ligand-core NLO-phores: a combined experimental and theoretical approach to the two-photon absorption and two-photon excited emission properties of small-ligated silver nanoclusters. Nanoscale 9 (3):1221-1228. doi:10.1039/C6NR07989J

18. Antoine R, Bonacic-Koutecky V (2018) Liganded silver and gold quantum clusters. Towards a new class of nonlinear optical nanomaterials. Springer, Cham,

19. Bonačić-Koutecký V (2017) Theoretical design of new class of optical materials based on small noble metal nanocluster-biomolecule hybrids and its potential for medical applications. Advances in Physics: X 2 (3):695-716. doi:10.1080/23746149.2017.1352458

20. Pyo K, Thanthirige VD, Kwak K, Pandurangan P, Ramakrishna G, Lee D (2015) Ultrabright Luminescence from Gold Nanoclusters: Rigidifying the $\mathrm{Au}(\mathrm{I})$-Thiolate Shell. Journal of the American Chemical Society 137 (25):8244-8250. doi:10.1021/jacs.5b04210

21. Jin R, Nobusada K (2014) Doping and alloying in atomically precise gold nanoparticles. Nano Research 7 (3):285-300. doi:10.1007/s12274-014-0403-5

22. Kreibig U, Vollmer M (1995) Optical Properties of Metal Clusters. Springer Verlag, Berlin Heidelberg,

23. Bonačić-Koutecky $V$, Veyret $V$, Mitrić $R$ (2001) Ab initio study of the absorption spectra of Agn ( $n=5-8)$ clusters. The Journal of Chemical Physics 115 (22):10450-10460.

doi:doi:http://dx.doi.org/10.1063/1.1415077

24. Fedrigo S, Harbich W, Buttet J (1993) Collective dipole oscillations in small silver clusters embedded in rare-gas matrices. Physical Review B 47 (16):10706-10715

25. Harbich W, Fedrigo S, Buttet J (1992) The Optical-Absorption Spectra of Small Silver Clusters ( $\mathrm{N}=$ 5-11) Embedded in Argon Matrices. Chem Phys Lett 195 (5-6):613-617. doi:10.1016/0009-

2614(92)85572-r

26. Harbich W, Fedrigo S, Buttet J, Lindsay DM (1991) Optical spectroscopy on size selected gold clusters deposited in rare gas matrices. Zeitschrift für Physik D Atoms, Molecules and Clusters 19 (4):157-159. doi:10.1007/bf01448280

27. Harbich W, Fedrigo S, Meyer F, Lindsay DM, Lignieres J, Rivoal JC, Kreisle D (1990) Deposition of mass selected silver clusters in rare gas matrices. The Journal of Chemical Physics 93 (12):8535-8543. doi:doi:http://dx.doi.org/10.1063/1.459291

28. Zheng J, Dickson RM (2002) Individual Water-Soluble Dendrimer-Encapsulated Silver Nanodot Fluorescence. Journal of the American Chemical Society 124 (47):13982-13983.

doi:10.1021/ja028282।

29. Richards Cl, Choi S, Hsiang J-C, Antoku Y, Vosch T, Bongiorno A, Tzeng Y-L, Dickson RM (2008) Oligonucleotide-Stabilized Ag Nanocluster Fluorophores. Journal of the American Chemical Society 130 (15):5038-5039. doi:10.1021/ja8005644 
30. Wu Z, Jin R (2010) On the Ligand's Role in the Fluorescence of Gold Nanoclusters. Nano Letters 10 (7):2568-2573. doi:10.1021/nl101225f

31. Diez I, Ras RHA (2011) Fluorescent silver nanoclusters. Nanoscale 3 (5):1963-1970.

doi:10.1039/c1nr00006c

32. Jin R (2010) Quantum sized, thiolate-protected gold nanoclusters. Nanoscale 2 (3):343-362. doi:10.1039/b9nr00160c

33. Brust M, Walker M, Bethell D, Schiffrin DJ, Whyman R (1994) Synthesis of thiol-derivatised gold nanoparticles in a two-phase Liquid-Liquid system. Journal of the Chemical Society, Chemical Communications (7):801-802. doi:10.1039/c39940000801

34. Jin R, Zeng C, Zhou M, Chen Y (2016) Atomically Precise Colloidal Metal Nanoclusters and Nanoparticles: Fundamentals and Opportunities. Chemical Reviews 116:10346-10413.

doi:10.1021/acs.chemrev.5b00703

35. Udayabhaskararao T, Pradeep T (2013) New Protocols for the Synthesis of Stable Ag and Au Nanocluster Molecules. J Phys Chem Lett 4 (Copyright (C) 2015 American Chemical Society (ACS). All Rights Reserved.):1553-1564. doi:10.1021/jz400332g

36. Schaaff TG, Knight G, Shafigullin MN, Borkman RF, Whetten RL (1998) Isolation and selected properties of a 10.4 kDa Gold: Glutathione cluster compound. J Phys Chem B 102 (52):10643-10646 37. Negishi Y, Nobusada K, Tsukuda T (2005) Glutathione-protected gold clusters revisited: Bridging the gap between gold(I)-thiolate complexes and thiolate-protected gold nanocrystals. J Am Chem Soc 127 (14):5261-5270

38. Negishi Y, Takasugi Y, Sato S, Yao H, Kimura K, Tsukuda T (2004) Magic-numbered Au-n clusters protected by glutathione monolayers $(n=18,21,25,28,32,39)$ : Isolation and spectroscopic characterization. Journal of the American Chemical Society 126 (21):6518-6519.

doi:10.1021/ja0483589

39. Kumar S, Bolan MD, Bigioni TP (2010) Glutathione-Stabilized Magic-Number Silver Cluster Compounds. Journal of the American Chemical Society 132 (38):13141-13143.

doi:10.1021/ja105836b

40. Zheng K, Yuan X, Goswami N, Zhang Q, Xie J (2014) Recent advances in the synthesis, characterization, and biomedical applications of ultrasmall thiolated silver nanoclusters. RSC Advances 4 (105):60581-60596. doi:10.1039/C4RA12054J

41. Zeng C, Chen Y, Das A, Jin R (2015) Transformation Chemistry of Gold Nanoclusters: From One Stable Size to Another. The Journal of Physical Chemistry Letters 6 (15):2976-2986.

doi:10.1021/acs.jpclett.5b01150

42. Yu Y, Chen X, Yao Q, Yu Y, Yan N, Xie J (2013) Scalable and Precise Synthesis of Thiolated Au1012, Au15, Au18, and Au25 Nanoclusters via pH Controlled CO Reduction. Chemistry of Materials 25 (6):946-952. doi:10.1021/cm304098x

43. Qian H, Eckenhoff WT, Zhu Y, Pintauer T, Jin R Total Structure Determination of ThiolateProtected Au-38 Nanoparticles. Journal of the American Chemical Society 132 (24):8280-+. doi:10.1021/ja103592z

44. Zhu M, Aikens CM, Hollander FJ, Schatz GC, Jin R (2008) Correlating the Crystal Structure of A Thiol-Protected Au25 Cluster and Optical Properties. Journal of the American Chemical Society 130 (18):5883-5885. doi:10.1021/ja801173r

45. Jin R (2015) Atomically precise metal nanoclusters: stable sizes and optical properties. Nanoscale 7 (5):1549-1565. doi:10.1039/C4NR05794E

46. Hamouda R, Bertorelle F, Rayane D, Antoine R, Broyer M, Dugourd P (2013) Glutathione capped gold AuN(SG)M clusters studied by isotope-resolved mass spectrometry. International Journal of Mass Spectrometry 335 (0):1-6

47. Chakraborty I, Pradeep T (2017) Atomically Precise Clusters of Noble Metals: Emerging Link between Atoms and Nanoparticles. Chemical Reviews 117 (12):8208-8271.

doi:10.1021/acs.chemrev.6b00769

48. Lu Y, Chen W (2015) Application of Mass Spectrometry in the Synthesis and Characterization of Metal Nanoclusters. Analytical Chemistry 87 (21):10659-10667. doi:10.1021/acs.analchem.5b00848 
49. Weerawardene KLDM, Aikens CM (2018) Origin of Photoluminescence of Ag25(SR)18Nanoparticles: Ligand and Doping Effect. The Journal of Physical Chemistry C 122 (4):2440-2447. doi:10.1021/acs.jpcc.7b11706

50. Weerawardene KLDM, Guidez EB, Aikens CM (2017) Photoluminescence Origin of Au38(SR)24 and Au22(SR)18 Nanoparticles: A Theoretical Perspective. The Journal of Physical Chemistry C 121 (28):15416-15423. doi:10.1021/acs.jpcc.7b01958

51. Weerawardene KLDM, Aikens CM (2016) Theoretical Insights into the Origin of

Photoluminescence of Au25(SR)18- Nanoparticles. Journal of the American Chemical Society 138 (35):11202-11210. doi:10.1021/jacs.6b05293

52. Fernando A, Weerawardene KLDM, Karimova NV, Aikens CM (2015) Quantum Mechanical Studies of Large Metal, Metal Oxide, and Metal Chalcogenide Nanoparticles and Clusters. Chemical Reviews 115 (12):6112-6216. doi:10.1021/cr500506r

53. Devadas MS, Kim J, Sinn E, Lee D, Goodson T, Ramakrishna G (2010) Unique Ultrafast Visible Luminescence in Monolayer-Protected Au25 Clusters. The Journal of Physical Chemistry C 114 (51):22417-22423. doi:10.1021/jp107033n

54. Wu ZK, Jin RC (2010) On the Ligand's Role in the Fluorescence of Gold Nanoclusters. Nano Lett 10 (7):2568-2573. doi:10.1021/nl101225f

55. Zheng J, Zhou C, Yu M, Liu J (2012) Different sized luminescent gold nanoparticles. Nanoscale 4 (14):4073-4083. doi:10.1039/C2NR31192E

56. Birge RR, Pierce BM (1979) A theoretical analysis of the two-photon properties of linear polyenes and the visual chromophores. The Journal of Chemical Physics 70 (1):165-178. doi:10.1063/1.437217 57. Przhonska OV, Webster S, Padilha LA, Hu H, Kachkovski AD, Hagan DJ, Van Stryland EW (2010) Two-Photon Absorption in Near-IR Conjugated Molecules: Design Strategy and Structure-Property Relations. In: Demchenko AP (ed) Advanced Fluorescence Reporters in Chemistry and Biology I: Fundamentals and Molecular Design. Springer Berlin Heidelberg, Berlin, Heidelberg, pp 105-147. doi:10.1007/978-3-642-04702-2_4

58. Hu Z, Jensen L (2017) Importance of double-resonance effects in two-photon absorption properties of Au25(SR)18. Chemical Science 8 (6):4595-4601. doi:10.1039/C7SC00968B 59. Bertorelle F, Moulin C, Soleilhac A, Comby-Zerbino C, Dugourd P, Russier-Antoine I, Brevet P-F, Antoine R (2018) Bulky counterions: enhancing the two-photon excited fluorescence of gold nanoclusters. ChemPhysChem 19:165-168. doi:10.1002/cphc.201701186

60. Russier-Antoine I, Bertorelle F, Kulesza A, Soleilhac A, Bensalah-Ledoux A, Guy S, Dugourd P, Brevet P-F, Antoine R (2016) Chiral supramolecular gold-cysteine nanoparticles: Chiroptical and nonlinear optical properties. Progress in Natural Science: Materials International 26:455-460. doi:http://dx.doi.org/10.1016/i.pnsc.2016.08.008

61. Russier-Antoine I, Bertorelle F, Vojkovic M, Rayane D, Salmon E, Jonin C, Dugourd P, Antoine R, Brevet P-F (2014) Non-linear optical properties of gold quantum clusters. The smaller the better. Nanoscale 6 (22):13572-13578. doi:10.1039/c4nr03782k

62. Makarov NS, Drobizhev M, Rebane A (2008) Two-photon absorption standards in the 550-1600 nm excitation wavelength range. Optics Express 16 (6):4029-4047. doi:10.1364/OE.16.004029 63. Kasha M (1950) Characterization of electronic transitions in complex molecules. Discussions of the Faraday Society 9 (0):14-19. doi:10.1039/DF9500900014

64. Van Steerteghem N, Van Cleuvenbergen S, Deckers S, Kumara C, Dass A, Hakkinen H, Clays K, Verbiest T, Knoppe $S$ (2016) Symmetry breaking in ligand-protected gold clusters probed by nonlinear optics. Nanoscale 8 (24):12123-12127. doi:10.1039/c6nr02251k

65. Le Guevel X, Trouillet V, Spies C, Li K, Laaksonen T, Auerbach D, Jung G, Schneider M (2012) High photostability and enhanced fluorescence of gold nanoclusters by silver doping. Nanoscale 4 (24):7624-7631. doi:10.1039/C2NR30653K

66. Brach K, Waszkielewicz M, Olesiak-Banska J, Samoc M, Matczyszyn K (2017) Two-Photon Imaging of 3D Organization of Bimetallic AuAg Nanoclusters in DNA Matrix. Langmuir 33 (36):8993-8999. doi:10.1021/acs.langmuir.7b00873 
67. Kindi HA, Mohamed A, Kajimoto S, Zhanpeisov N, Horino H, Shibata Y, Rzeznicka II, Fukumura H (2018) Single bovine serum albumin molecule can hold plural blue-emissive gold nanoclusters: A quantitative study with two-photon excitation. Journal of Photochemistry and Photobiology A: Chemistry. doi:https://doi.org/10.1016/j.jphotochem.2018.02.029

68. Raut SL, Shumilov D, Chib R, Rich R, Gryczynski Z, Gryczynski I (2013) Two photon induced luminescence of BSA protected gold clusters. Chemical Physics Letters 561 (Supplement C):74-76. doi:https://doi.org/10.1016/j.cplett.2013.01.028

69. Porret E, Sancey L, Martín-Serrano A, Montañez MI, Seeman R, Yahia-Ammar A, Okuno H, Gomez F, Ariza A, Hildebrandt N, Fleury J-B, Coll J-L, Le Guével X (2017) Hydrophobicity of Gold Nanoclusters Influences Their Interactions with Biological Barriers. Chemistry of Materials 29 (17):7497-7506. doi:10.1021/acs.chemmater.7b02497

70. Shen D, Henry M, Trouillet V, Comby-Zerbino C, Bertorelle F, Sancey L, Antoine R, Coll J-L, Josserand V, Guével XL (2017) Zwitterion functionalized gold nanoclusters for multimodal near infrared fluorescence and photoacoustic imaging. APL Materials 5 (5):053404.

doi:10.1063/1.4977203 
metal nanoclusters, 2, 4, 5, 6

B

bio-imaging applications, 1, 13, 19

Bulky counterions, 17, 24

C

charge transfer excited state, 8

confocal microscopy, 18

E

Endogenous non-linear optics, 2

$\mathbf{F}$

fluorescein, 15

$\mathbf{G}$

gel electrophoresis, 6

Glutathione, 6

K

Kasha's rule, 17

\section{M}

Multi-photon optics, 2

\section{$\mathbf{P}$}

proteins, 2, 20

push-pull dyes, 3

Q

quantum chemistry methods, 4

\section{S}

scaffold, 6

Second Harmonic Generation, 2

silver doping, 20, 24

susceptibility, 2

\section{$\mathbf{T}$}

Thiols, 6

Third Harmonic Generation, 2

time-dependent density functional theory, 1, 8

TPA cross section, 10, 12

TPE cross-sections, 15

transition dipole moments, 10, 11, 13

transition energies, 10

Two-Photon Excitation Fluorescence, 2

mass spectrometry, 6, 7, 23

medium polarization, 2 